Revue des patrimoines et plasticiens (XXe-XXle siècles)

\title{
Architectes et plasticiens dans la conception et la réalisation du campus de la faculté des Lettres et Sciences humaines de Montpellier
}

Architects and plastic artists in the design of the humanities faculty of the university at Montpellier

\section{Yvon Comte}

\section{OpenEdition}

Journals

Édition électronique

URL : http://journals.openedition.org/insitu/14748

DOI : $10.4000 /$ insitu. 14748

ISSN : $1630-7305$

Éditeur

Ministère de la culture

Référence électronique

Yvon Comte, «Architectes et plasticiens dans la conception et la réalisation du campus de la faculté des Lettres et Sciences humaines de Montpellier », In Situ [En ligne], 32 | 2017, mis en ligne le 27 juillet 2017, consulté le 19 avril 2019. URL : http://journals.openedition.org/insitu/14748; DOI : 10.4000/ insitu. 14748

Ce document a été généré automatiquement le 19 avril 2019

\section{c) (†)}

In Situ Revues des patrimoines est mis à disposition selon les termes de la licence Creative Commons Attribution - Pas d'Utilisation Commerciale - Pas de Modification 4.0 International. 


\title{
Architectes et plasticiens dans la conception et la réalisation $\mathrm{du}$ campus de la faculté des Lettres et Sciences humaines de Montpellier
}

\author{
Architects and plastic artists in the design of the humanities faculty of the \\ university at Montpellier
}

Yvon Comte

1 L'intégration de la création artistique plastique à l'architecture est particulièrement prise en compte dans la conception et la réalisation de la partie « littéraire » (alors «faculté des Lettres et Sciences humaines ») du campus universitaire de Montpellier. Il s'agit en effet de l'œuvre la plus aboutie du vaste projet engagé dans les années 1960, qui porte sur les deux facultés, Sciences d'une part et Lettres d'autre part. La conception de cette dernière relève d'un véritable objet architectural et artistique à part entière, associant complètement l'œuvre d'art à la construction et à l'aménagement de l'espace. La commande au titre du $1 \%$ artistique fait appel à des noms célèbres (Victor Vasarely), moins connus (Robert Pillods) ou plus locaux (Fernand Michel et Albert Dupin). Ces créations de 1964 à 1966 précèdent celles de la faculté des Sciences qui se placent entre 1969 et $1970^{1}$.

2 L'ambitieux programme immobilier des universités de Montpellier des années 1960 fait l'objet très tôt, d'un avant-projet établi sous la direction de l'architecte marseillais René Egger², personnalité importante qui s'est fait connaître dans le domaine de la construction scolaire et universitaire ${ }^{3}$. En effet, architecte en chef des Bâtiments civils et Palais nationaux, il est aussi conseiller technique de l'Éducation nationale de 1942 à 1968. À Marseille, Egger travaille déjà avec les grands noms de l'art contemporain comme Picasso au lycée de Marseilleveyre, Vasarely à la faculté des Sciences Saint-Jérôme, César au lycée Est, ou encore Amado, Pignon et Zadkine. Son architecture monumentale reste sobre, inspirée des principes du Mouvement moderne, privilégiant le béton armé mais 
dans un esprit méditerranéen, faisant la part belle aux espaces d'agoras, de patios et utilisant la végétation locale et l'eau'. Pour le projet d'ensemble universitaire nord de Montpellier, René Egger travaille à l'origine avec, comme architecte d'opération, le cabinet montpelliérain de Jean de Richemond ${ }^{5}$. Ce cabinet compte alors parmi ses collaborateurs l'architecte Philippe Jaulmes ${ }^{6}$, qui est aussi passionné par le concept de la cinétique intégré à l'art. C'est avec lui, bientôt associé avec Jean-Claude Deshons dans le cadre de leur agence commune ${ }^{7}$, que l'aventure architecturale des facultés de Montpellier se poursuit (fig. 1).

Figure 1

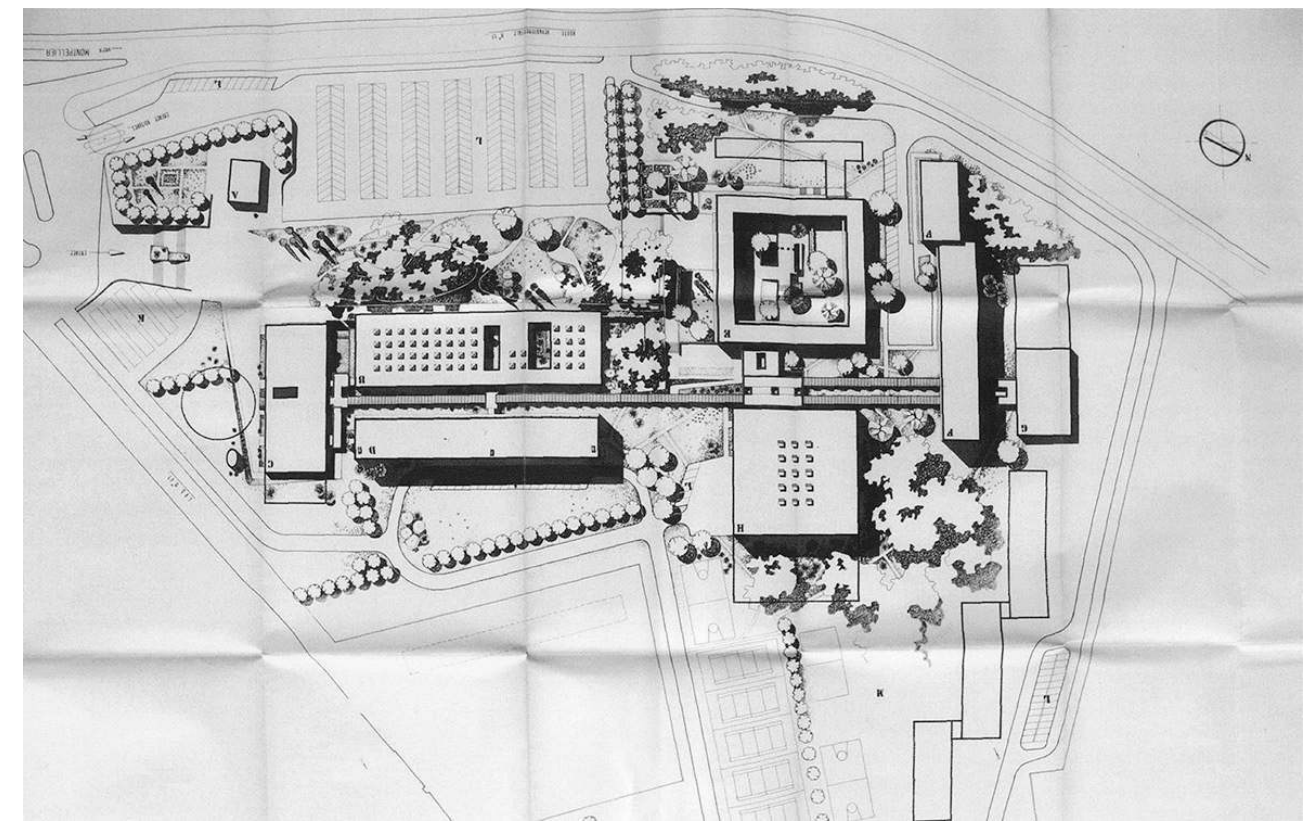

Plan général de la partie « Lettres et Sciences humaines » du campus de Montpellier établi en 1963 par l'agence de Philippe Jaulmes et Jean-Claude Deshons. Archives de l'UPV, fonds ancien 9 F.A, direction de la logistique immobilière.

Phot. Yvon Comte, DRAC Occitanie, CRMH, Montpellier, 2011. ( ) DRAC Occitanie.

Philippe Jaulmes insiste beaucoup sur sa formation initiale à l'École nationale supérieure des beaux-arts de Paris, qui marque durablement sa production, avant même qu'il ne s'oriente vers l'architecture. Il travaille sur un nouveau concept associant architecture et cinéma dans une démarche complémentaire pour appréhender l'espace non seulement fixe mais aussi dans sa mobilité. En 1954, son diplôme porte sur le projet d'une salle hémisphérique pour projections avec objectif «fish-eye » à $360^{\circ}$. En 1958, il brevète le procédé "Panrama, cinéma, temps, espace ", qu'il met en œuvre dans un bâtiment expérimental qu'il construit à Clapiers, près de Montpellier, abritant une vaste coupole inclinée de 12 mètres de diamètre ${ }^{8}$. En 1963 il fonde la société « les Ateliers du cinéma total ». L'évolution des techniques et de l'informatique permet aujourd'hui d'aller encore plus loin dans cette voie. Dès les années 1950, le développement de sa théorie et de ses expérimentations s'appuie en partie sur le travail cinétique de l'Opart de Vasarely mais s'enracine aussi dans les recherches des peintres de toutes les époques sur l'anamorphose, puisqu'il essaie d'adapter la représentation visuelle de la réalité aux deux dimensions d'une toile ou d'un écran dans une perspective dynamique où spectateur et œuvre interagissent par le déplacement du corps ou du regard. L'architecte cherche ainsi 
à donner au public une place d'acteur en ajoutant une dimension d'impondérable, variable pour chacun. Dans ses premiers films, il s'inspire notamment des monuments de Montpellier, comme le château d'eau du Peyrou (œuvre majeure du grand maître d'œuvre montpelliérain $\mathrm{du} \mathrm{xVIII}^{\mathrm{e}}$ siècle Jean-Antoine Giral), pour restituer ces volumes géométriques rigoureux basés sur les modules de l'hexagone et du trigone. «L'architecte peut ainsi concrétiser ses rêves, les faire partager à d'autres, faire évoluer ses projets vers une création authentique, par le choix de ses aller-retour » écrit-il en 1986 dans la revue Carré bleu $u^{9}$. En effet, pour Jaulmes, la représentation de l'espace architectural par l'image en deux dimensions est complètement inapte à figurer une réalité que l'image totale, par ses courbes reportées sur la sphère, mise en mouvement par le cinéma, peut désormais restituer grâce à l'informatique (fig. 2).

Figure 2

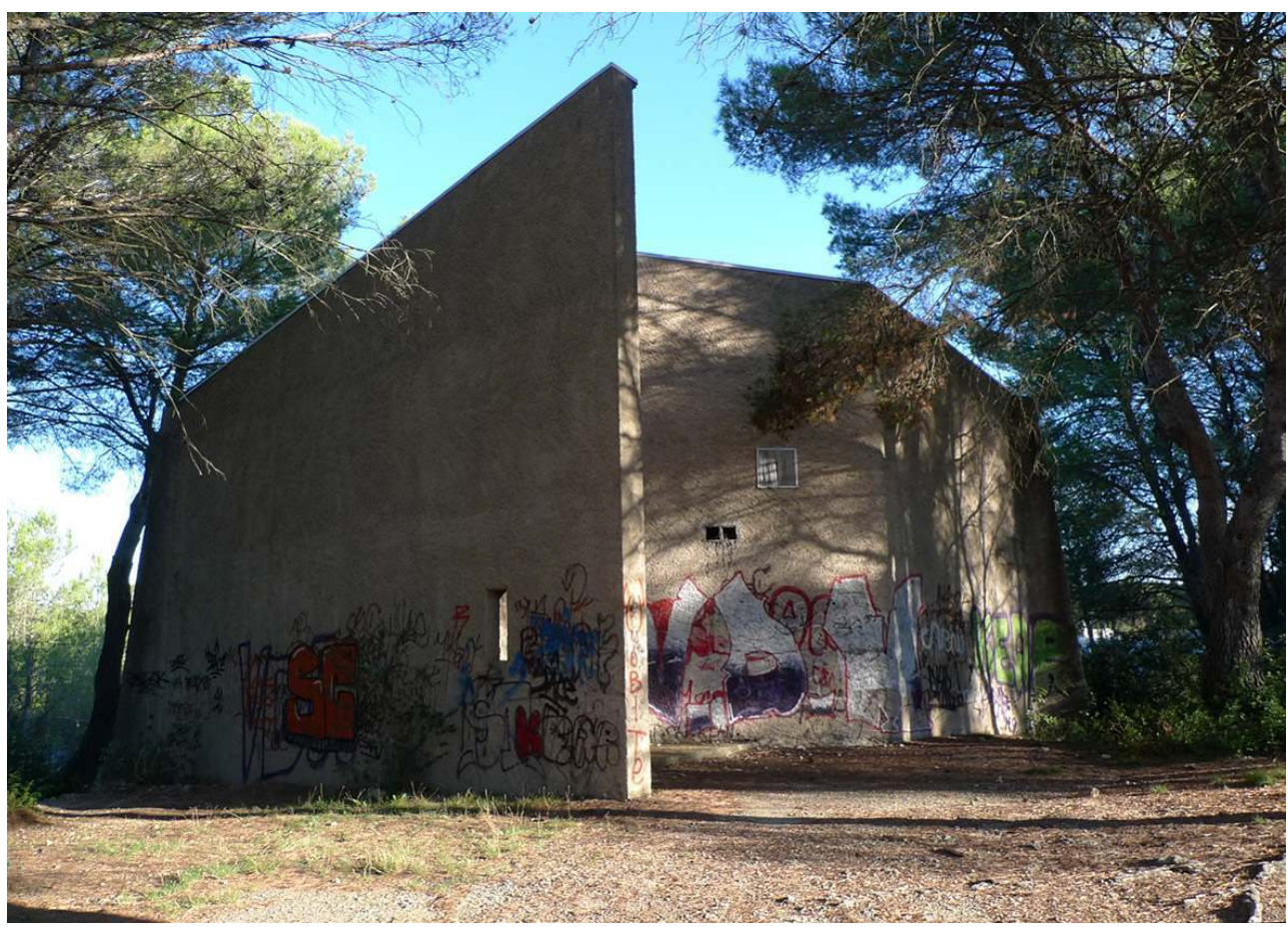

Vue d'ensemble du cinéma expérimental « Panrama » par Philippe Jaulmes.

Phot. Yvon Comte, DRAC Occitanie, CRMH, Montpellier, 2011. (c) DRAC Occitanie.

4 Associé à la maîtrise d'œuvre de la faculté des Sciences, Philippe Jaulmes réalise notamment le bâtiment administratif et la bibliothèque des Sciences. Il peut y introduire plusieurs créations artistiques majeures, prenant ainsi ses distances par rapport à l'esprit encore très traditionnel de Jean de Richemond qui dirige le projet : il est, par exemple, un des premiers à rejeter les toitures de tuiles pour des toits-terrasses plus contemporains. En réaction avec les modes de construction de l'époque, il adopte une démarche esthétique héritée de sa formation des Beaux-Arts. Dans ses prises de parole ${ }^{10}$, il insiste sur son goût pour la création contemporaine qui l'amène à travailler avec des artistes du courant de l'art en mouvement. Ses influences architecturales assumées sont le Mouvement moderne et Le Corbusier, mais aussi Jean Prouvé pour l'attention qu'il porte à l'économie de la construction, à la rapidité d'exécution et à l'utilisation de l'aluminium industriel. Il considère fondamental que le $1 \%$ artistique permette au maître d'œuvre des constructions publiques de choisir les artistes avec qui il peut collaborer, dans un 
dialogue dynamique. Cela le conduit à se rapprocher d'abord de Victor Vasarely et d'Yvaral, puis de Yaacob Agam et de Pol Bury, à qui il commande des œuvres pour les deux facultés de Montpellier. Systématiquement, il impulse cette dynamique de création artistique contemporaine dans la construction nouvelle, mettant l'accent sur le lien qui unit l'architecte et les artistes ${ }^{11}$.

Pour la faculté des Sciences, dont la première pierre est posée en 1961 et la construction achevée pour la rentrée de 1964, René Egger est très présent et l'influence de Jean de Richemond reste prépondérante: on construit des bâtiments fonctionnels et économiques, constitués de longues barres à la géométrie symétrique et uniforme, évoquant les grands ensembles d'habitation. Cependant, au cœur du complexe universitaire, un espace naturel est préservé et aménagé en arboretum (il est aujourd'hui peu perceptible). Au centre de ce secteur, Philippe Jaulmes élève un groupe de deux blocs carrés : le bâtiment administratif (dit aussi «bâtiment de la Présidence ») s'organisant autour d'un atrium et la bibliothèque, plus singulière, où l'architecte introduit des concepts esthétiques qu'il développera plus $\operatorname{tard}^{12}$ (fig. 3).

Figure 3

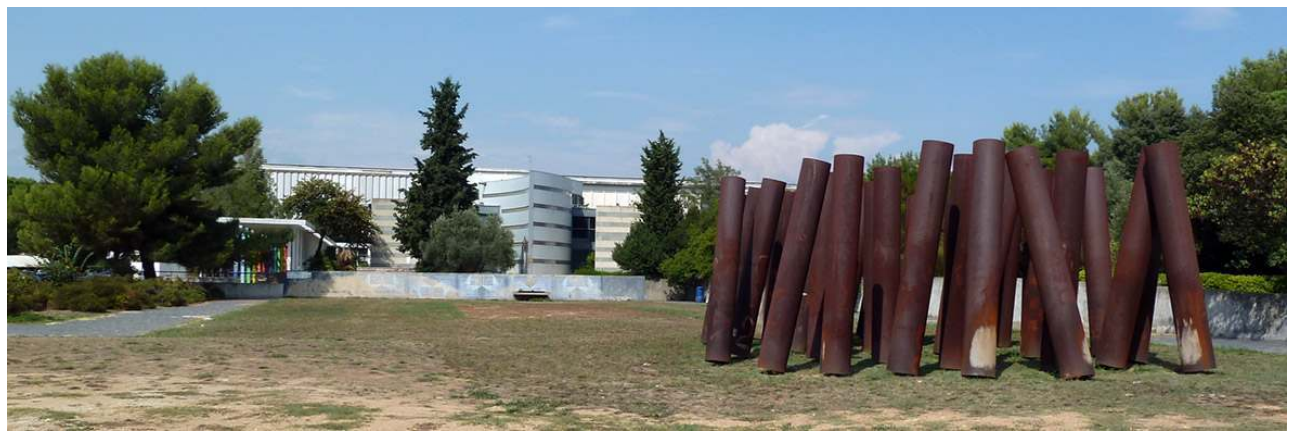

Euvres du campus de la faculté des Sciences de Montpellier : Méditation en action, sculpture en cylindres d'acier COR-TEN par Pol Bury (1922-2005) et, en arrière-plan, mur peint de motifs cinétiques par Yvaral (1934-2002).

Phot. Yvon Comte, DRAC Occitanie, CRMH, Montpellier, 2011. (c) DRAC Occitanie.

6 Les études préliminaires de la faculté des Lettres, cosignées par Egger et l'agence JaulmesDeshons en octobre $1961^{13}$, comportent un « rapport explicatif d'architecte » qui aborde la qualité paysagère du programme et qui souligne que l'ensemble universitaire se situe «sur un terrain bordé au nord de très belles zones boisées». Dans le projet, la topographie, en léger dénivelé, est bien prise en compte, de même que l'insertion dans le paysage avec, notamment, des vues lointaines vers la ville : « Il est intéressant de signaler qu'il est en quelque sorte orienté vers les jardins du Pérou [place royale du Peyrou, ndlr] et qu'il bénéficie d'une très belle vue dégagée sur la campagne montpelliéraine ». Par ailleurs, les architectes sont soucieux d'aboutir à une certaine cohérence entre les deux unités (Sciences et Lettres) du vaste programme universitaire: "Les axes directeurs principaux de la première faculté ont été respectés dans l'implantation de la seconde. » La volonté de respecter la nature des lieux est également affirmée. Cependant, la réalisation finale, dans laquelle les plasticiens jouent un rôle prégnant, sera assez différente du premier schéma. En effet, en 1961, l'Institut d'art, avec ses musées des moulages et d'archéologie, n'est pas encore prévu. Par ailleurs, l'esthétique des bâtiments rappelle les sobres élévations de Fernand Pouillon plutôt que les profils contemporains qui seront 
finalement adoptés (l'avant-projet définitif, accompagné d'un nouveau plan de masse, est mis au point en avril 1962).

La fonctionnalité reste la première exigence du programme et les bâtiments doivent être en adéquation avec les besoins spécifiques d'une université, comporter des infrastructures appropriées à l'enseignement magistral et à la pédagogie universitaires. Le lien entre ces éléments se fait par des galeries de circulation et des lieux d'échanges privilégiant la dynamique de déambulation, comme les atriums et les espaces verts. Par ailleurs, pour la partie du projet concernant les "Lettres", à la différence de celle concernant les «Sciences", l'agence montpelliéraine intervient en tant que véritable maître d'œuvre ; René Egger ne joue qu'un rôle secondaire dans sa conception et sa mise en œuvre. Jaulmes et Deshons s'y affirment notamment dans l'intégration de la décoration monumentale (fig. 4).

Figure 4

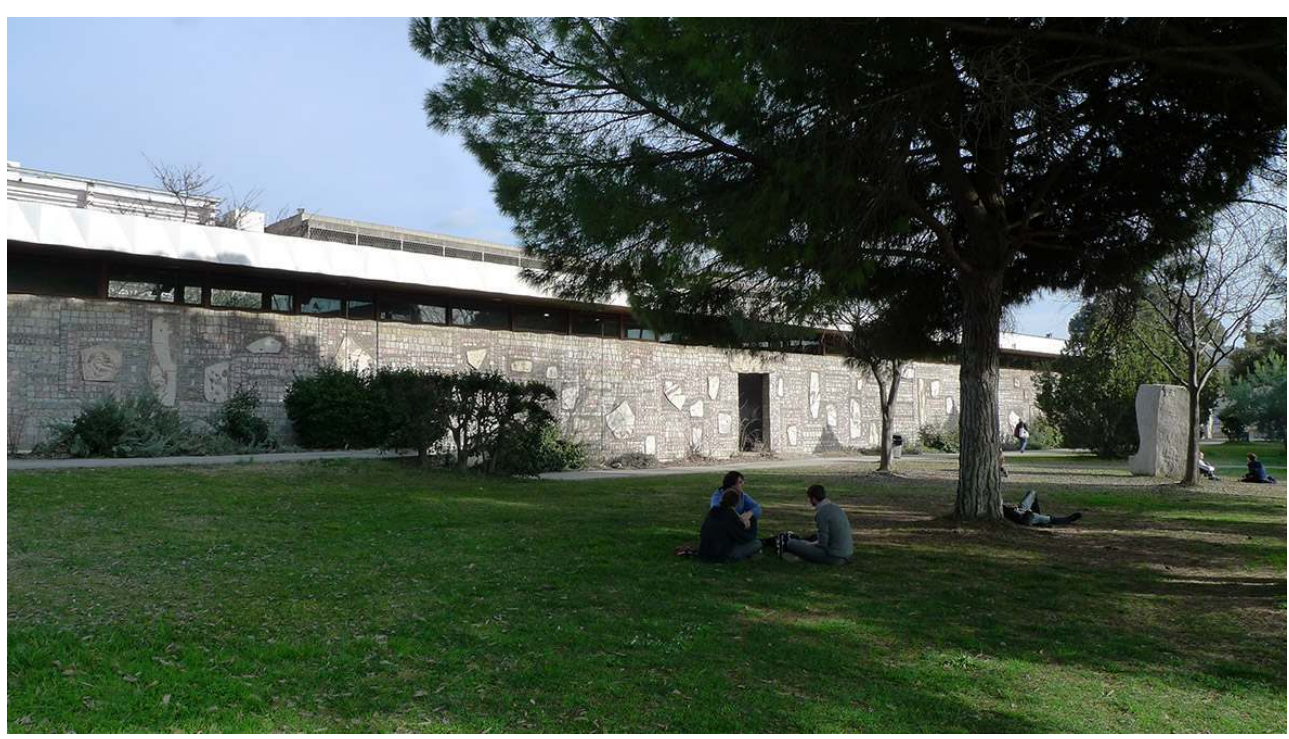

Ambiance naturelle du parc et vue générale du bâtiment d'Art et d'archéologie du campus de la faculté des Lettres et Sciences humaines, avec le mur dit « cyclopéen » d'Albert Dupin (1910-2005) en arrièreplan.

Phot. Yvon Comte, DRAC Occitanie, CRMH, Montpellier, 2011. (c) DRAC Occitanie.

Le « rapport décoration » de l'avant-projet mérite à ce titre d'être analysé avec attention. Le parti d'ensemble y est détaillé, révélant une intention didactique. Ce texte permet de mesurer la métamorphose de la sensibilité artistique des maîtres d'œuvre qui évoluent vers une réflexion et une abstraction intellectuelles plus avant-gardistes. À l'origine, « les architectes proposent comme thème général à soumettre aux artistes pressentis la légende d'Icare ", car " ce thème permet des développements plastiques très variés dans l'espace et par son caractère universel souligne la fonction d'échanges culturels d'une faculté des Lettres.» Les auteurs du rapport précisent même: «la première tranche destinée à la propédeutique servirait de cadre à l'illustration de la lente germination de l'esprit dans la gangue originelle, à la patiente élaboration du « concept » à partir de la " matière inerte » ». C'est là que naît, semble-t-il, la première idée de ce qui deviendra la création d'Albert Dupin. En effet, « pour concrétiser cette idée les architectes envisagent la construction de l'élévation principale du musée des Moulages sous la forme d'un mur en pierre cyclopéenne destiné à créer par son échelle et sa "présence » un véritable 
décor d'accueil et d'accompagnement. » Il est étonnant de constater à quel degré de détail s'attachent les architectes dans les orientations qu'ils donnent: "au milieu de cette masse éminemment physique, seraient enchâssées et judicieusement réparties des petites surfaces en céramique hautes en couleurs. »

L'intégration de la couleur, question qui traverse toutes les époques, principalement et notamment dans l'architecture religieuse et publique, a souvent été abordée par le biais du vitrail. Pour la faculté des Lettres et Sciences humaines de Montpellier, Philippe Jaulmes fait appel à Robert Pillods, artiste atypique qui a évolué du dessin d'illustration à la grande composition monumentale en passant par la peinture de chevalet ${ }^{14}$. Au cours de la deuxième partie de sa vie, Robert Pillods se consacre à la réalisation de grands décors architecturaux mobilisant différentes techniques. Les premiers sont conçus pour des temples ou des églises de l'est de la France, comme les dalles de verre qu'il imagine pour l'église protestante d'Ostheim (Haut-Rhin) reconstruite par Georges Hirlemann et René Schmitt entre 1958 et 1960. René Schmitt est alors architecte des bâtiments de France en Lozère, d'où est originaire l'épouse de Robert Pillods. Il favorise ainsi l'accès à la commande de l'artiste en Languedoc-Roussillon. Robert Pillods y réalise notamment un mur de verre à la maison protestante du Lazaret à Sète (Hérault) et les vitraux des baies du temple à Vialas (Lozère) $(1960,1967)$ (fig. 5).

Figure 5

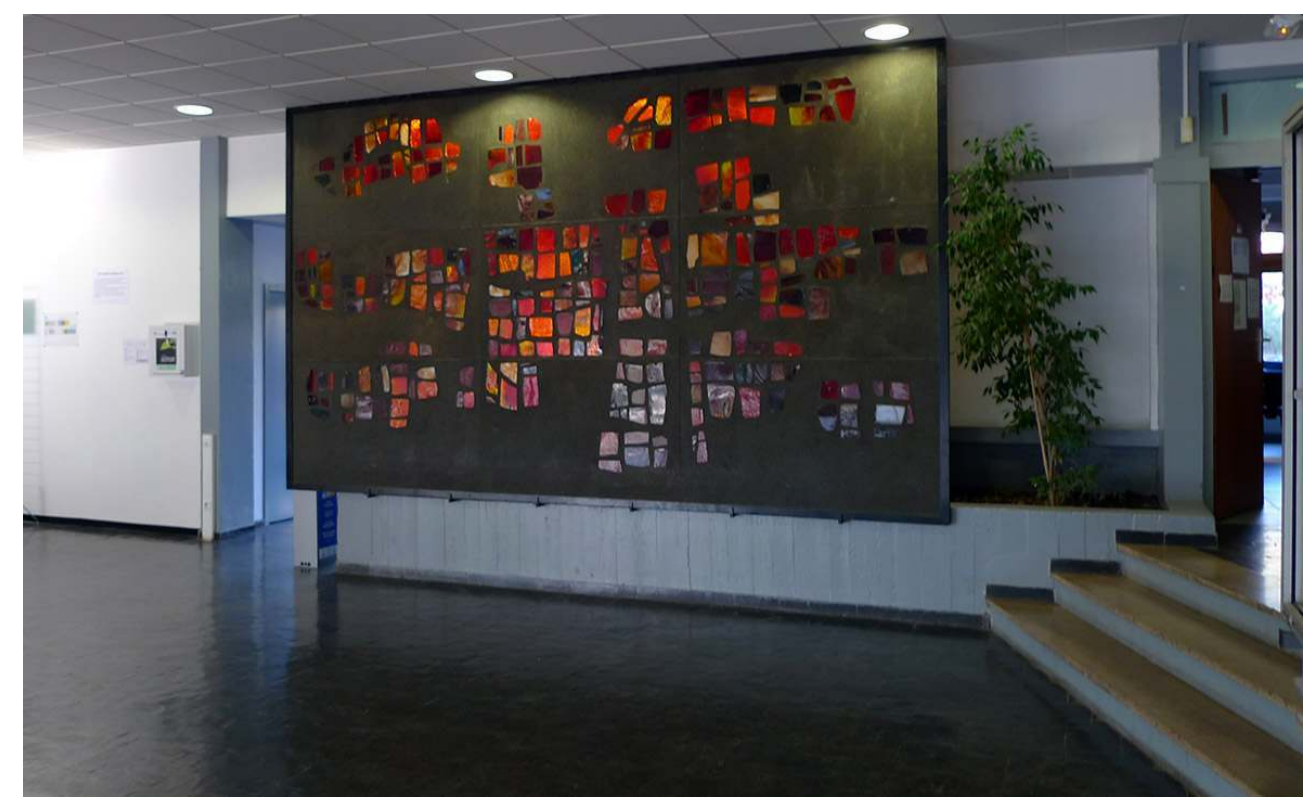

Panneau dit «Tapisserie de résine et de dalles de verre » de Robert Pillods (1908-1990) dans le hall du bâtiment administratif de la faculté des Lettres et Sciences humaines.

Phot. Yvon Comte, DRAC Occitanie, CRMH, Montpellier, 2011. @ DRAC Occitanie.

De plus, René Schmidt est un ami de Philippe Jaulmes, à qui il recommande Robert Pillods pour créer un décor intégré à la faculté des Lettres de Montpellier. L'artiste propose de réaliser deux grands panneaux qu'il nomme " tapisseries de résine et de dalles de verre ", ne souhaitant pas qualifier ces œuvres de "vitraux ». En effet, le verre y est inséré dans un support de résine polyester coulée artisanalement par l'artiste, de façon à ce que le relief et la matière opaque acquièrent un sens graphique. Mais il conçoit aussi ces œuvres comme des sculptures, en traitant les deux faces de façon complémentaire mais 
différente, variant formes et effets de transparence. Chaque panneau rectangulaire (2,5 x 3,7 mètres) est constitué de neuf modules incrustés de pavés translucides dessinant une composition abstraite indépendante du cloisonnement. L'une de ses œuvres, installée dans le grand hall fermé du bâtiment administratif, est dans les dominantes rouges; l'autre, dans les dominantes bleues, formait un écran dans le passage ouvert sous le bâtiment des Licences (bâtiments B et C) dans l'axe principal de la faculté. Il a depuis été déplacé et il est désormais abrité à l'étage d'un des nouveaux bâtiments (bâtiment $\mathrm{D}$ ). Le panneau intérieur est signé « Pillods » et porte la marque des ateliers parisiens Barillet ${ }^{15}$, célèbres depuis les années 1920, et désormais animés par Jean Barillet qui a travaillé avec les plus grands plasticiens du $\mathrm{xx}^{\mathrm{e}}$ siècle tels que Fernand Léger, Le Moal et Bazaine à Audincourt, Braque à Varengeville-sur-Mer, Manessier à Hem ou bien encore Moutier en Suisse. Très attaché au mur-écran de la faculté des Lettres et Sciences humaines de Montpellier, Robert Pillods s'est montré très affecté par sa dégradation, se proposant même, peu avant son décès, de venir le restaurer. Pour lui, l'installation de l'œuvre n'était pas anodine puisque son intérêt réside dans la perception que peut en avoir le public lors de ses déplacements et selon les variations de lumière, ce qui intéresse aussi Philippe Jaulmes dans sa réflexion sur le dynamisme de l'art (fig. 6).

Figure 6

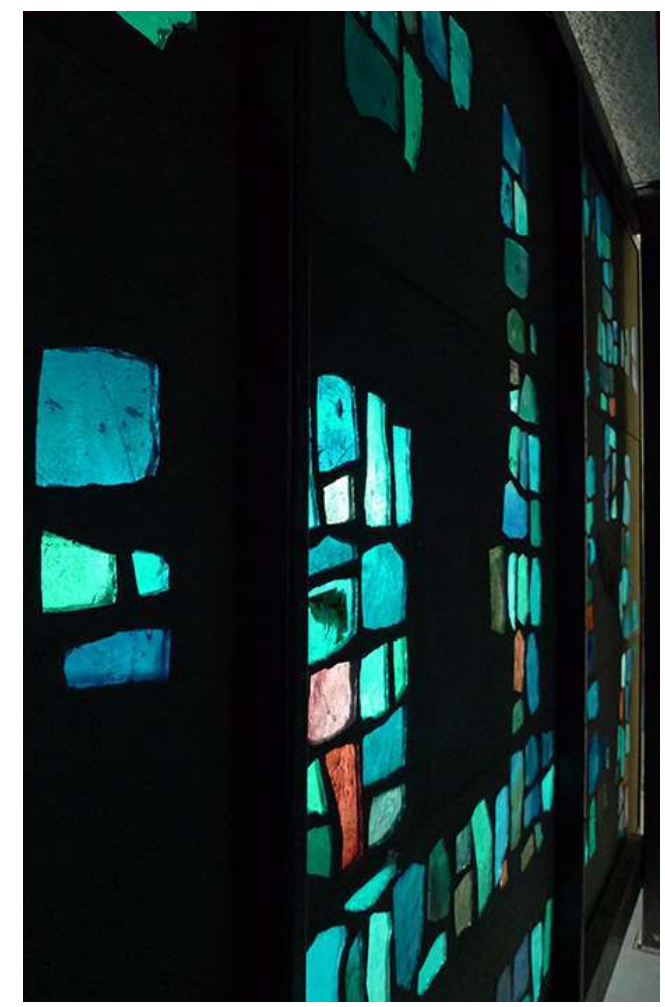

Détail de la « Tapisserie de résine et de dalles de verre » de Robert Pillods (1908-1990) installée initialement en transition des bâtiments de licence de la faculté des Lettres et Sciences humaines. Phot. Yvon Comte, DRAC Occitanie, CRMH, Montpellier, 2011. ( ) DRAC Occitanie.

Les architectes de la faculté insistent d'ailleurs sur la place essentielle accordée au traitement de l'entrée de l'université: elle doit être majestueuse et accueillante, largement ouverte par des degrés dégagés, dont l'effet ascendant est souligné par la grande grille mobile de Vasarely. Si Egger avait déjà collaboré en Provence avec ce ténor 
de l'Op'art (art optique), à Montpellier, c'est Philippe Jaulmes qui s'adresse directement à Victor Vasarely pour la commande de la grande grille de la faculté des Lettres. En effet, la démarche de l'artiste inspire grandement les réflexions de l'architecte qui parvient alors à des conclusions assez parallèles aux siennes. Dans sa jeunesse, dès 1929, Vasarely s'était d'abord intéressé au Bauhaus, qu'il avait découvert à l'académie Muhëly de Budapest, avant de se rapprocher du constructivisme. Ce n'est qu'après s'être réfugié à Paris, en 1930, qu'il s'oriente vers un graphisme plus pur et pose les bases de son cinétisme abstrait. Ses compositions restent encore ancrées dans les rapports d'ombre et de lumière qui jouent, comme Jaulmes tentera de le mettre en œuvre trente ans plus tard dans son architecture, avec la perspective et les déformations optiques. Zebra (1940) est l'aboutissement de cette recherche, véritable manifeste de l'op'art, art abstrait propre à Vasarely, utilisant un vocabulaire restreint de couleurs et de formes. Ses recherches sur le mouvement trouvent une traduction éditoriale dans le Manifeste jaune ${ }^{16}$ où il affirme que seul le regard est créateur, et non la composition. Il y développe aussi le thème de la démocratisation d'un art social accessible à tous (fig. 7). Il collabore alors avec son fils Jean-Pierre Vasarely, dont le nom d'artiste est Yvaral, auteur également d'œuvres sur le campus de la faculté des Sciences de Montpellier. Dans le domaine de l'intégration architecturale, Vasarely travaille dès 1954 avec l'architecte Carlos Villanueva à la Cité universitaire de Caracas au Venezuela ${ }^{17}$ puis crée de nombreuses autres œuvres monumentales. Dans sa manière d'appréhender l'architecture, il prend en compte non seulement l'espace mais aussi les structures et les matériaux, notamment industriels, envisageant l'art et la technique de construction dans un rapport de symbiose. Il veut établir un lien organique entre l'art et l'architecture, avec des œuvres réalisées directement dans le corps de l'édifice.

Figure 7

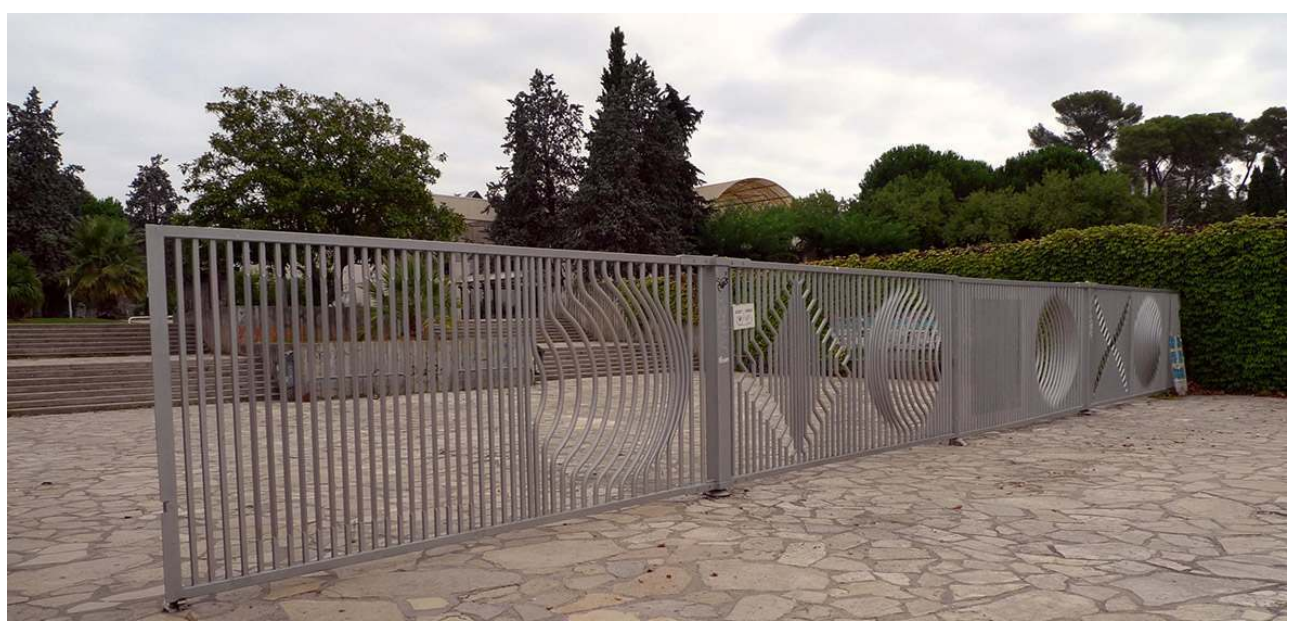

Grille monumentale de Victor Vasarely (1906-1997) située à l'entrée de la faculté des Lettres et Sciences humaines

Phot. Yvon Comte, DRAC Occitanie, CRMH, Montpellier, 2011. (c) DRAC Occitanie.

En Languedoc-Roussillon, l'œuvre majeure de Victor Vasarely est la grille du portail d'entrée de la faculté des Lettres et Sciences humaines de Montpellier. Celle-ci est le résultat d'une longue maturation de ses expériences, nourries par ailleurs de l'usage d'éléments préfabriqués issus de procédés industriels afin d'accéder à une échelle de création monumentale. La grille prend la forme d'une longue structure pivotante en 
tubulure métallique à effet cinétique reprenant le thème de SIR-RIS, récurrent dans l'œuvre de l'artiste, dont le nom fait référence à la brillante Sirius chère à Vasarely pour son symbolisme d'étoile double. Ce motif associe des cercles, des croix et des losanges dans une distorsion de lignes et d'ombres créant une illusion d'optique. Traités sur un plan en $2 \mathrm{D}$, les cercles apparaissent bombés et sont perçus en tridimensionnel selon le mouvement du spectateur et les jeux d'éclairage. Cet effet est obtenu par des barreaux métalliques plus ou moins rapprochés et courbés, produits par le remarquable travail du ferronnier qui a concrétisé la vision de Vasarely ${ }^{18}$ (fig. 8). La volonté de Victor Vasarely est donc de suggérer en trompe-l'œil un relief mouvant, tantôt concave, tantôt convexe, provoquant la participation du spectateur qui perçoit jusqu'au gonflement induit par la déformation des lignes, c'est-à-dire des images qui s'échappent du plan pour suggérer des volumes.

Figure 8

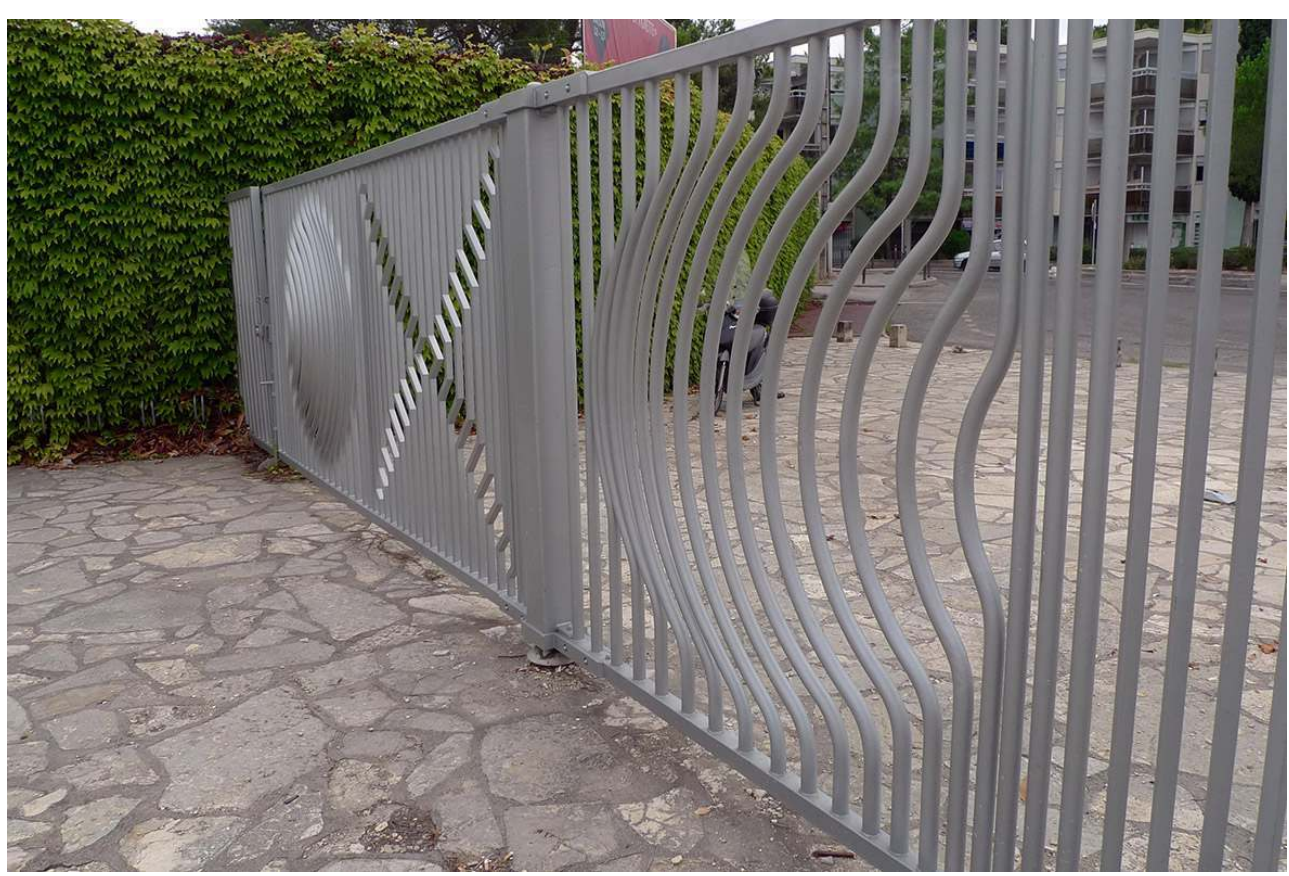

Détail de l'effet 3 D de la grille d'entrée par Victor Vasarely (1906-1997).

Phot. Yvon Comte, DRAC Occitanie, CRMH, Montpellier, 2011. @ DRAC Occitanie.

13 Alors que les travaux de la faculté des Lettres et Sciences humaines auraient dû débuter dès 1962 (pour une livraison des premiers bâtiments en 1964), le Conseil général des bâtiments de France demande la révision du projet dans le sens d'une "expression originale ». Il faut attendre décembre 1963 pour que le projet d'exécution définitif soit approuvé. Un lieu consacré à l'art et à l'archéologie, qui n'était pas prévu lors du tout premier programme, est ajouté grâce notamment au doyen Hubert Gallet de Santerre, professeur d'archéologie antique. Sa définition évolue vers un véritable Institut d'art et d'archéologie regroupant un bâtiment spécifique destiné à donner à la collection des moulages un cadre adapté à la mesure de son importance historique et pédagogique, spécifiquement conçu pour elle, auquel on adjoint un local consacré à l'archéologie avec un musée des antiquités (fig. 9). 


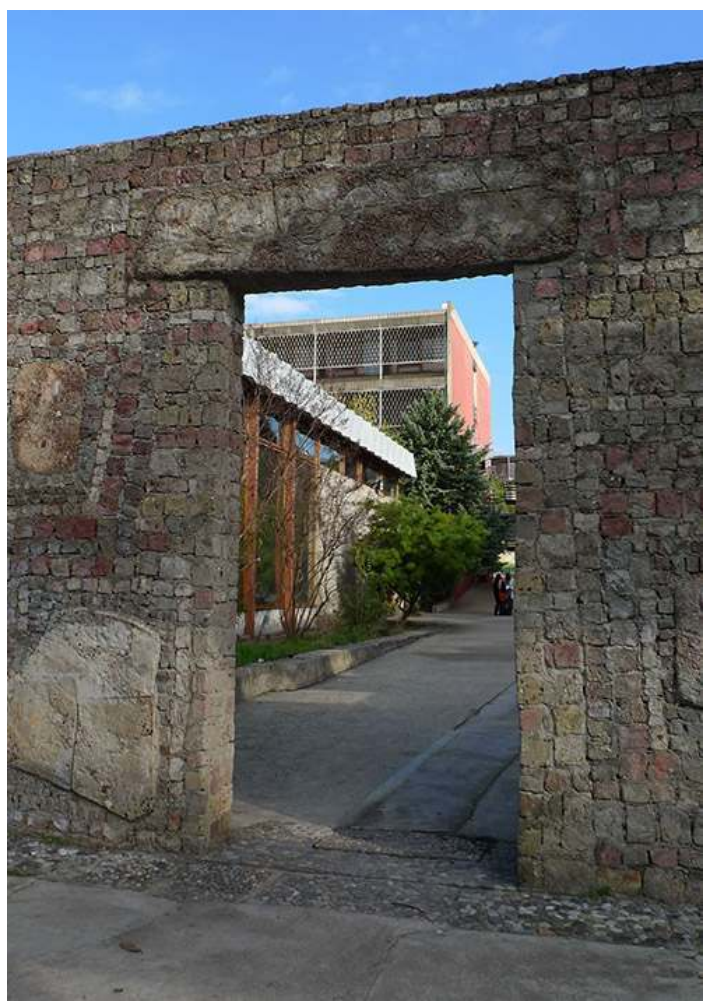

Porte d'accès du musée des Moulages percée dans le mur « cyclopéen » d'Albert Dupin (1910-2005). Phot. Yvon Comte, DRAC Occitanie, CRMH, Montpellier, 2011. ( ) DRAC Occitanie.

Les artistes locaux prennent aussi une part importante au projet avec, notamment, un grand mur que l'architecte imagine en appareil cyclopéen, évocation archéologique des remparts de la Grèce mycénienne. La composition plastique non figurative exécutée par le sculpteur Albert Dupin ${ }^{19}$ est bien inspirée de cette idée. Dupin est un ami proche de Jean-Claude Deshons qui le décrit comme « le paysan Dupin, acharné à pousser son sillon, modeleur de sites, créateur d'espaces ${ }^{20}$. Ce dernier a suivi les cours des beaux-arts à Toulouse de 1926 à 1928, s'est formé aussi à la lithographie puis a travaillé pour un atelier d'ébénisterie. À partir de 1931, il sculpte le bois, s'essaie à la céramique comme beaucoup d'artistes de son temps et, en 1944, réalise sa première sculpture en taille directe. Bien qu'essentiellement sculpteur, il peint et dessine. Le geste du dessinateur a d'ailleurs pour lui une importance primordiale, même s'il s'en libère lors de l'exécution de ses sculptures. À partir de 1937-1938, il expose à Paris et, en 1945, il présente ses compositions sculpturales au Salon d'automne dont il devient sociétaire. En 1950 et 1951, il participe au premier Salon de la jeune sculpture au musée Rodin et, en 1954, au Salon de Mai. Dès 1942, il avait côtoyé certains peintres du "groupe Frédéric Bazille» comme Gabriel Couderc, Camille Descossy, Georges Dezeuze, Jean Milhau, mais aussi Gérard Calvet, Pierre Fournel, Jean-Raymond Bessil. Il est invité ensuite aux manifestations du groupe "Montpellier-Sète" rassemblant en 1964, autour de François Desnoyer et Camille Descossy, des artistes comme Maurice-Élie Sarthou. Tous ont comme point commun leur enracinement méridional, bien qu'ils n'appartiennent pas aux mêmes "familles" esthétiques. À partir de 1962, Albert Dupin s'intéresse à l'intégration de la sculpture à l'architecture. Il s'oriente vers des compositions murales et des bas-reliefs non figuratifs (fig. 10). 
Figure 10

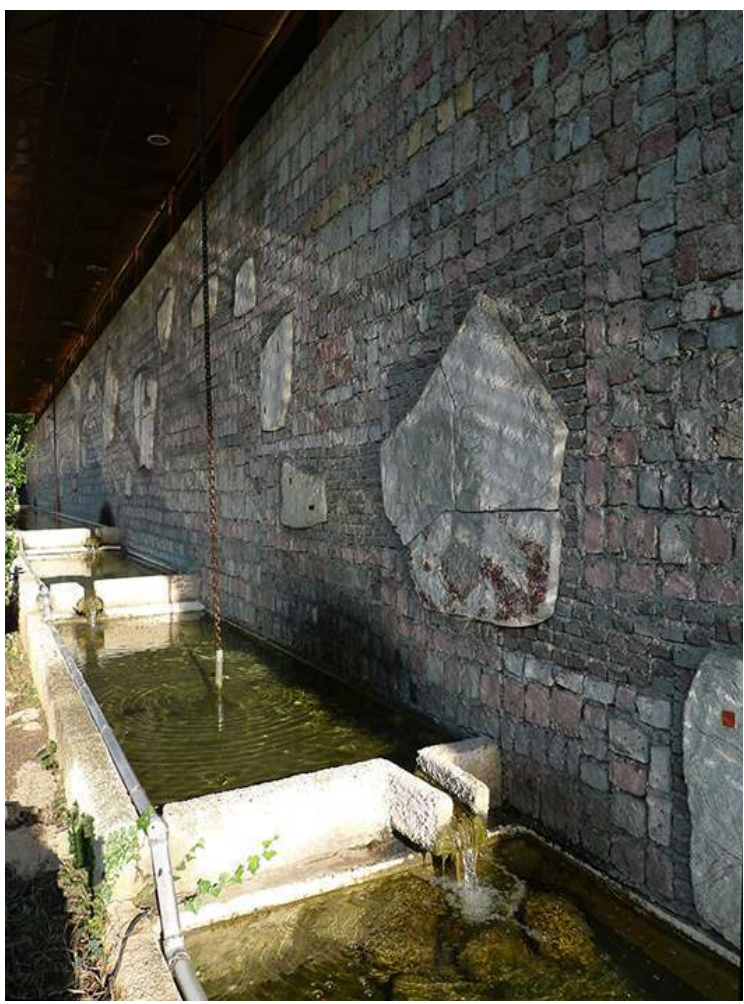

Mur « cyclopéen » d'Albert Dupin (1910-2005) avec effet de cascade le long du bâtiment d'Art et d'Archéologie.

Phot. Yvon Comte, DRAC Occitanie, CRMH, Montpellier, 2011. (c) DRAC Occitanie.

Une de ses œuvres monumentales majeures est le mur de la faculté des Lettres de Montpellier ; peut-être devrait-on plutôt parler de véritable muraille, pour désigner cette partie intégrante du bâti puisqu'elle forme la façade ouest du musée des Moulages de la faculté des Lettres et Sciences humaines de Montpellier. Résultant d'une commande publique passée en 1964 dans le cadre du $1 \%$ artistique, cette composition s'étend sur 120 mètres de long. Bien que non figurative, elle relève d'une démarche inverse à celle de l'abstraction, dans le sens où elle est faite de matériaux bruts, terres cuites et fragments de céramiques émaillées, pierres volcaniques et graviers de la région du Salagou. Ces éléments hétérogènes présentent des aspects irréguliers, incertains, souvent indéterminés. Ils sont mêlés, noyés dans des panneaux de béton réalisés en atelier par Albert Dupin avec son fils Jean-Pierre, puis assemblés sur site par des maçons. Ils composent une longue façade protégée par un débord généreux de toiture et éclairée par les reflets d'un petit canal, en dialogue avec l'espace naturel préservé au centre de la faculté. Dans l'espace universitaire montpelliérain, Albert Dupin réalise aussi un mursculpture en terre cuite, rythmé de motifs en relief accrochant la lumière, pour la façade extérieure courbe de la salle de rencontres (aujourd'hui "Trioletto») de la résidence universitaire du Triolet (1964) et un ensemble de sculptures pour l'atrium du bâtiment administratif de la faculté des Sciences, sur le thème des Sept signes de vie (1970) (fig. 11) 


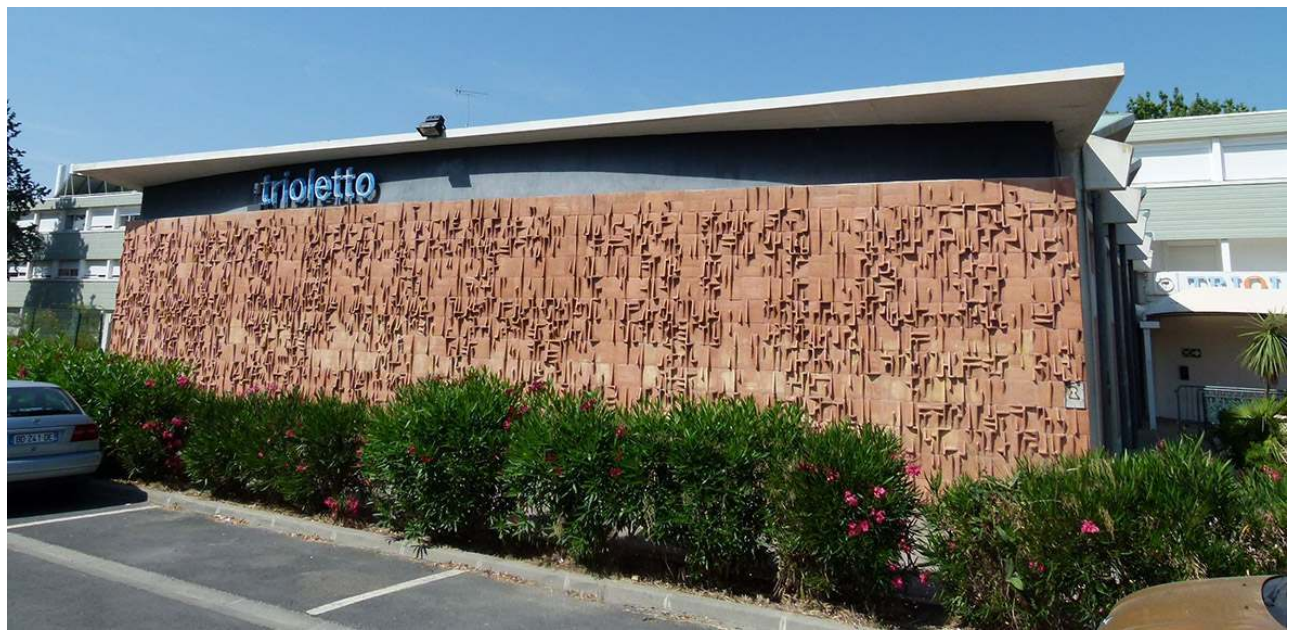

Mur décoré en terre-cuite d'Albert Dupin (1910-2005) à la résidence universitaire du Triolet.

Phot. Yvon Comte, DRAC Occitanie, CRMH, Montpellier, 2011. (c) DRAC Occitanie.

À la bibliothèque universitaire de la faculté des Lettres et Sciences humaines, si le programme fonctionnel est assez contraignant, Philippe Jaulmes tient néanmoins à insérer une création plastique originale. Pour cela, il s'adresse à Fernand Michel ${ }^{21}$, personnage atypique, figure locale de l'art brut et ami de Jean-Claude Deshons. Il lui confie la réalisation d'un grand panneau mural non figuratif pour le hall d'entrée que l'artiste traite en fort relief, à partir de coques et de coudes de zinc ouverts de 5,4 X 3,5 mètres. Il n'est pas anodin que Fernand Michel intervienne en ce lieu qui est le symbole de la richesse littéraire de l'université. En effet, sa formation de relieur d'art (il est «meilleur ouvrier de France ») le conduit à collaborer avec de nombreux écrivains, poètes et éditeurs pour illustrer de gravures des recueils de poésie surréaliste comme Les variations typographiques de Jean Vodaine sur deux poèmes de Raymond Queneau ${ }^{22}$. Chez Fernand Michel, la pratique de la gravure préfigure la découverte du zinc, matériau pour lequel il se passionne. Il raconte comment, vers 1962, cherchant des fragments d'objets chargés de pouvoir évocateur sur une décharge à Palavas-les-Flots, il trouve une plaque de zinc dont les oxydations lui rappellent certaines de ses gravures. À partir de là, il réalise des "zingueries", assemblages, souvent de grande dimension. Il réalise des paysages constitués de plaques de zinc découpées et simplement passées sous la presse du relieur, ou bien juxtaposées pour composer un panorama poétique. Fernand Michel n'utilise aucun oxydant ni colorant car le zinc est « un matériau relativement poreux qui se chargeait de toutes les oxydations des autres métaux à son contact ainsi que du calcaire et des macérations du bois ou des déchets organiques ». Fernand Michel réalise aussi des "pièces ludiques", érotiques, humoristiques et surréalistes. Elles sont composées à partir d'objets de métal récupérés, détournés, fragmentés comme des globes de protection électrique des toitures d'Alsace que l'artiste utilise pour représenter des baigneuses plantureuses, Vénus callipyges ou bonnes sœurs. Poète, il accompagne souvent ses œuvres de vers de René Char ou de Saint-John Perse. Il crée également un bestiaire inspiré du Voyage en Grande Garabagne, en hommage à Henri Michaux ${ }^{23}$. À Montpellier, outre la faculté des Lettres et Sciences humaines, l'hôtel de région et l'IUT donnent à voir de monumentaux panneaux muraux d'aluminium ou en zinc cuivré de Fernand Michel (fig. 12). 
Figure 12

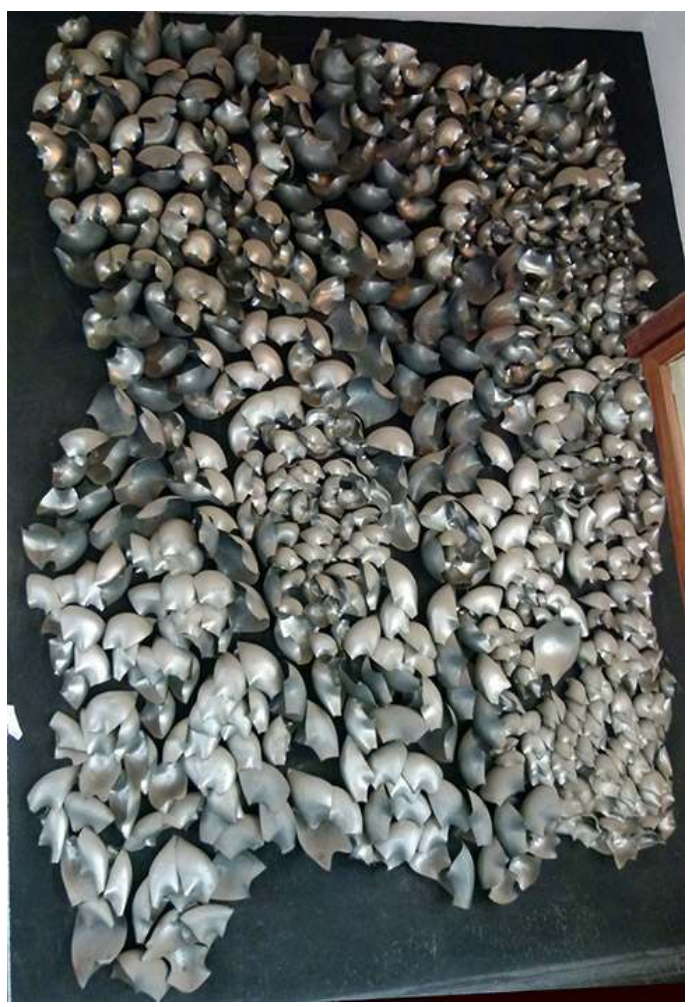

Panneau en coques de zinc de Fernand Michel (1913-1999) du hall de la bibliothèque universitaire de la faculté des Lettres et Sciences humaines.

Phot. Yvon Comte, DRAC Occitanie, CRMH, Montpellier, 2011. (c) DRAC Occitanie.

À la faculté des Lettres et Sciences humaines de Montpellier, les architectes, très sensibles à la création contemporaine et fins connaisseurs des mouvements artistiques contemporains, choisissent de lier intimement la création plastique avec l'architecture, selon une logique d'intégration. L'art cinétique de Victor Vasarely y entre en écho avec l'ambition architecturale de Philippe Jaulmes qui, au travers d'une architecture indissociable du mouvement qui la parcourt, entendait mettre en lien l'humain et son environnement. La lumière et l'image animée, engagée par Philippe Jaulmes dans son projet de cinéma total, trouve un écho à la faculté des Lettres où le visiteur, même actuel, peut se laisser guider par le jeu de la lumière mouvante et des formes stables d'une architecture sobre, fonctionnelle mais originale et vivante. La qualité relationnelle que Jean-Claude Deshons a su nouer avec des artistes locaux plus secrets et pleins d'originalité, en prise directe avec la matière brute, a permis d'introduire dans cette architecture d'abord fonctionnelle, une part de sensibilité poétique propre au terroir. Alors que maintes réalisations contemporaines de la faculté des Lettres et Sciences humaines de Montpellier associant intimement art et architecture sont encore, dans le meilleur des cas, ignorées mais plus souvent, ont été déjà bafouées ou même détruites, la labellisation du campus au titre du patrimoine $d u x^{e}$ siècle laisse espérer que soit conservé ce cadre original dans lequel art et architecture forment un tout harmonieux. 


\section{NOTES}

1. - Entre 1969 et 1970, sculpture d'Albert Dupin, installation de Yaacov Agam, panneau mural de Victor Vasarely, puis de 1972 à 1974, tapisserie de François Desnoyer, décors muraux d'Yvaral et mobile de Pol Bury ; le cadran solaire de la cour d'honneur, dû à François Rouan et Pierre Parsus, a malheureusement disparu.

2. - René Egger (1915-2016). D'abord associé à Fernand Pouillon, avec qui il réalise d'importants programmes de construction à Marseille et à Aix-en-Provence, il est le principal artisan du Marseille de l'après-guerre, en participant avec Pouillon à la reconstruction du quartier du Vieux Port puis seul, surtout à partir de 1953. Il forme avec Gaston Defferre un « couple » de bâtisseurs de 1955 à 1985, modelant la ville et son paysage urbain (EGGER, René. Avec Gaston Deferre. Marseille : Éditions européennes de Marseille-Provence, 2001).

3. - MARANTZ, Éléonore. «René Egger ou la réinvention de l'architecture universitaire ». Dans COMPAIN-GAJAC, Catherine (dir.). Les campus universitaires. Architecture et urbanisme, histoire et sociologie, état des lieux et perspectives. Perpignan : PUP, 2014, p. 131-146.

4. - Une dizaine de grandes réalisations de René Egger est labellisée « Patrimoine $d u x^{e}$ siècle». Face à la pression démographique, il bâtit vite, partout et avec peu de moyens. En moins de dix ans, il édifie à Marseille 150 « écoles Egger », des constructions standardisées et, en l'absence de normes, il crée des modèles de groupes scolaires, écoles maternelles, primaires, collèges. Il est l'auteur de grands ensembles universitaires, principalement à Marseille mais aussi à Nice et Toulouse-Rangueil. Il réalise l'école des beaux-arts et d'architecture de Marseille (1965-1967-1969) où il s'inspire des campus américains. Non moins novatrice est la faculté de Médecine (1955-1958). À ce programme universitaire est liée la construction des grands hôpitaux. 5. - Jean Meschinet de Richemond (1904-1983), neveu et successeur de l'architecte montpelliérain Edmond Leenhardt, architecte des bâtiments publics et palais nationaux, professeur d'architecture à Montpellier. On lui doit d'importants ensembles des années 1950 et 1960, notamment sociaux ou de l'éducation nationale comme architecte du rectorat dans les années 1950. Il est, à ce titre, l'auteur de l'Institut de botanique conçu pour abriter l'exceptionnel herbier de la faculté de Pharmacie (AD Hérault, fonds Jean de Richemond $61 \mathrm{~J}$ ).

6. - Philippe Jaulmes est né à Montpellier en 1927 (AD Hérault, fonds Philippe Jaulmes $200 \mathrm{~J}$; archives de l'université Paul-Valéry (UPV), fonds ancien $9 \mathrm{~F}$. A, direction de la logistique immobilière).

7. - L'agence d'architecture de Philippe Jaulmes et Jean-Claude Deshons construit les principaux immeubles publics de Montpellier dans les années 1960 à 1980 et travaille sur la faculté des Lettres, les cités et restaurants universitaires (Vert-Bois et Le Triolet) entre 1963 et 1966. En 1964, une des principales réalisations de l'agence est l'usine modèle d'IBM. Jaulmes et Deshons conçoivent aussi le CNRS et surtout, en 1973-1975, l'hôtel de ville de Montpellier dont la construction avait été décidée en 1969 par le maire François Delmas. Cet immeuble aux façades de verre implanté dans le quartier alors en pleine urbanisation de la ZAC du Polygone (dont Jaulmes et Deshons sont les architectes coordonnateurs) enjambe la voie ferrée et permet ainsi l'extension de la ville vers l'est; sa reconversion fait aujourd'hui l'objet d'une réflexion. On doit aussi à Jaulmes et Deshons la direction régionale des télécommunications en 1978, les locaux de la DDE, de l'ONF et, plus tard, le réaménagement de l'hôpital Saint-Éloi (1992). À Saint-Clémentde-Rivière, l'agence conçoit la clinique de la Lironde selon un plan à trame triangulaire. Bien que l'essentiel de la production de l'agence reste publique, elle répond aussi à des commandes privées (par exemple maison Colombier, au nord de Montpellier, 1968). 
8. - Le Panrama fait actuellement l'objet d'un permis de démolir dans le cadre d'un vaste projet de promotion immobilière mais il vient d'être inscrit au titre des monuments historiques et proposé au classement par la commission régionale du patrimoine et des sites du Languedoc-Roussillon. En 1981, le procédé est présenté dans de plus amples proportions à l'Espace Gaité à Paris (quatre ans avant la géode de la Villette) (JAULMES, Philippe. Cinéma, temps et espace, introduction au panrama, procédé de cinéma total. préf. Abel Gance. Montpellier : chez l'auteur, 1964 ; Id. L'écran total, pour un cinéma sphérique. Paris : Lherminier, 1981).

9. - Carré bleu, revue internationale d'architecture, $\mathrm{n}^{\circ} 2-3,1986$. Philippe Jaulmes contribue à ce numéro consacré à la création architecturale et informatique avec un article intitulé «Une application de l'informatique pour aller plus loin dans la troisième dimension » (p. 38-39).

10. - Voir notamment les entretiens privés accordés à l'auteur en 2011 et la conférence publique sur le Panrama donnée par MM. Hubert Corbin et Yvon Comte à la DRAC à Montpellier le 14 juin 2016 dans le cadre du « mois de l'Architecture ».

11. - HANDEL-CLÉMENTIN, Nathalie. Le $1 \%$ à Montpellier, université Paul-Valéry, université des Sciences et techniques du Languedoc. DEA histoire de l'art. Montpellier : UPV Montpellier, 1997.

12. - Les œuvres y sont toutes visibles dans cet espace restreint allant de l'entrée, avec le mobile de cylindres dressés au milieu du parterre vert, en acier COR-TEN, de Pol Bury, alternant parties fixes et instables, intitulé "méditation en action » jusqu'au cœur administratif avec son patio agrémenté d'une sculpture en ronde-bosse d'Albert Dupin, en passant par le mur peint de Yvaral (Jean-Pierre Vasarely) auquel fait écho le décor géométrique des grilles de clôture du campus dû à Philippe Jaulmes et à son dessinateur Paul Bouthier. La grande tapisserie de François Desnoyer et l'installation de Yaacob Agam Huit plus un en mouvement, baguettes colorées éclairées (déposées), plus fragiles, sont abritées à l'intérieur. Vasarely réalise le bardage en feuilles d'aluminium, à l'origine colorées, de la façade de la bibliothèque, irrégulièrement soulevées pour accrocher la lumière et créer des ombres et des reflets mouvants, décor particulièrement en adéquation avec la recherche cinétique de l'architecte.

13. - COMTE, Yvon, PALOUZIÉ, Hélène, PINCHON, Jean-François. Le campus de la faculté des Lettres et Sciences humaines de Montpellier, une création architecturale et artistique des années 1960. Montpellier : DRAC Languedoc-Roussillon, 2012.

14. - Robert Pillods (1908-1990) est natif du Doubs, où il travaille tout jeune comme dessinateur industriel pour les usines Peugeot. Adulte, il découvre la peinture régionaliste et fonde une Société des artistes peintres du pays de Montbéliard où il expose d'abord ses paysages et portraits, avant de s'installer comme artiste à Paris en 1951. Il est présent au Salon des indépendants, au Salon d'automne et dans plusieurs galeries à Paris, Bâle, Casablanca, Barcelone. Évoluant alors vers un dessin plus stylisé, il se fait connaître notamment pour ses illustrations au fusain de la Bible (Images de l'Ancien Testament, Images de l'Évangile). Toujours à la recherche de nouveaux moyens expressifs, il s'intéresse à Cézanne, au cubisme et se dirige assez vite vers une abstraction colorée évoquant le vitrail par la fragmentation des plans et de la lumière. Il reçoit une dizaine de commandes publiques au titre du $1 \%$ artistique, ce qui lui permet de toucher à d'autres formes d'art (par exemple, à l'école élémentaire Crusem à Saint-Avold (Moselle), où il conçoit, en 1966, une sculpture en blocs de béton) (MÜLLER, Marc. « Robert Pillods, artiste peintre ». Bulletin de la Société d'émulation de Montbéliard, n¹20, Montbéliard, 1997, p. 159-199).

15. - ARCHIERI, Jean-François, NEBOUT, Cécile (dir.). Atelier Louis Barillet, maître verrier. Paris : Éditions 15, Square de Vergennes, 2005.

16. - VASARELY, Victor. Le Manifeste jaune. Paris : galerie Denise René, 1955.

17. - MARANTZ, Éléonore. «La cité universitaire de Caracas, un idéal impossible pour le Groupe Espace?». Dans GAY, Diana (dir.). L'été 54 à Biot. Architecture, Formes, couleur. Paris : RMN, 2016, p. 92-95. 
18. - Cette œuvre est publiée dans le catalogue de l'Exposition triennale de Milan en 2007 («Un plasticien al servizio della Città » (ARRADON, Monique, VASARELY, Victor. «Vasarely: un plasticien dans la cité ». La nouvelle critique, $n^{\circ} 45$, Librairie nouvelle, 1971) en parallèle avec la grille-sculpture cinétique de lames d'aluminium du musée de Jérusalem exécutée la même année, et publiée dans la monographie intitulée Vasarely (VASARELY, Victor. Vasarely. Introd. Marcel J oray. Neuchâtel : Éditions du Griffon, 1965, sq.).

19. - Albert Dupin (1910-2005) passe son enfance à Lodève et s'installe définitivement dans le Lodévois (Octon) dès 1957. Il y bâtit son atelier où il passe la dernière partie de sa vie. Ses autres murs décoratifs et sculptures pour le $1 \%$ sont nombreuses : Mutuelle du bâtiment à Paris (1966), lycée technique Saint-Dié, mur de soutènement (projet 1965, réalisation 1969) en terre cuite, lycée Mermoz à Montpellier (1971, détruit), CEG de Port-Vendres (projet 1968, réalisation 1974) en béton, CET Saint-Jean-de-Védas (1976), lycée mixte de Lodève : fontaine (1973), cité scolaire de Bastia (1975), CES Jean-Moulin à Sète : sculpture Née de l'Etang (1980). Plusieurs de ses œuvres sont conservées dans les musées de l'Hérault: musée Fleury de Lodève, musée Fabre de Montpellier (qui possède quelques archives de l'artiste), musée régional d'Art contemporain de Sérignan, musée Paul-Valéry à Sète, fondation Desnoyer à Saint-Cyprien. Plusieurs études universitaires lui sont consacrées.

20. - CHATELET, Laurence. Albert Dupin, sculpteur. Mémoire de maîtrise, histoire de l'art. Montpellier : université Paul-Valéry, 1989. RAMIO, Céline. Albert Dupin, quand la lumière devient forme. Mémoire de maîtrise, histoire de l'art. Montpellier : université Paul-Valéry, 1999.

21. - Fernand Michel (1913-1999) est originaire d'Alsace mais il est actif à Montpellier où il commence à travailler dans une usine de poterie avant de devenir relieur d'art. Il entre alors dans le monde de l'art brut dans le sillage de Jean Dubuffet et Alphonse Chave. Ses œuvres sont exposées à la galerie des Mages d'Alphonse Chave à Vence (haut lieu de l'art brut dans les années 1960 et 1970), et au Petit musée du bizarre de Candide (alias Serge Tekielski) à Lavilledieu (Ardèche). Il participe aux expositions « Les Indomptables de l'art » à Besançon en 1986, et « Art Brut et Cie » à la Halle Saint-Pierre en 1995-1996. Son œuvre est présentée dans la collection de La Fabuloserie à Dicy (en Bourgogne, collection d'Alain Bourbonnais, œuvres d'autodidactes, art singulier ou hors les normes. Elle fait l'objet d'expositions régulières à Castelnau-le-Lez (ARPAC, Association régionale pour la promotion de l'art contemporain) depuis 1979. Jean-Claude Deshons a été président de l'association des Amis de Fernand Michel (HANDEL-CLEMENTIN, Nathalie. Fernand Michel, artiste zingueur. DEA, histoire de l'art. Montpellier: université PaulValéry, 1997; ALLAMEL, Frédéric. Imaginaires zincifêres, variations autour d'un artiste-zingueur Fernand Michel. Montpellier : ADABS, 2011.

22. - QUENEAU, Raymond, VODAINE, Jean, MICHEL, Fernand. Deux poèmes, « Dire » et "Trop tard ». Variations typographiques sur deux poèmes de Raymond Queneau. Paris : Éditions Dire, 1964.

23. - MICHAUX, Henri. Voyage en Grande Garabagne. Paris : Nouvelle revue française, 1936.

\section{RÉSUMÉS}

Le campus de la faculté des Lettres et Sciences humaines (aujourd'hui « université Paul-Valéry ») Montpellier-III est la création la plus aboutie des projets de constructions universitaires montpelliéraines des années 1960. L'ensemble comprenant à l'origine la faculté des Sciences est dirigé par l'architecte en chef marseillais René Egger qui travaille ici en collaboration avec les architectes d'opération montpelliérains Jean-Claude Deshons et Philippe Jaulmes. Ces derniers 
évoluent, pour la faculté des Lettres, vers des solutions plus esthétiques avec la mise en œuvre monumentale de la création artistique intégrée ( $1 \%$ culturel»), liant architectes et artistes. Déjà Egger avait collaboré avec Victor Vasarely qui conçoit à Montpellier la grande grille d'entrée du campus. Des artistes locaux interviennent aussi, notamment Albert Dupin avec le mur dit « cyclopéen » des bâtiments d'art et d'archéologie.

The campus of the Faculty of Letters and Humanities at today's Paul-Valéry UniversityMontpellier III is the most successful creation amongst the Montpellier university buildings constructed during the 1960s. The campus originally included the Faculty of Science designed by René Egger, the celebrated architect from Marseille. He worked there together with the project architects Jean-Claude Deshons and Philippe Jaulmes, from Montpellier. For the Faculty of Letters and Humanities, these last two architects evolved towards more aesthetic solutions integrating artistic creation (the artistic $1 \%$ ), associating architects and artists. Previously, Egger had collaborated with the kinetic Op art artist Victor Vasarely, whose work is beautifully illustrated at Montpellier by the great entrance railing of the campus. Other local artists intervened too in this programme, such as Albert Dupin with his so called 'cyclopean wall' designed for the Art and Archaeology Museum.

\section{INDEX}

Keywords : René Egger, Jean-Claude Deshons, Philippe Jaulmes, Victor Vasarely, Albert Dupin, Robert Pillods, Fernand Michel, Yvaral (Jean-Pierre Vasarely), Yaacob Agam, Pol Bury, university, university architecture, campus, Op-art, optical Art, kinetic Art, Montpellier

Mots-clés : René Egger, Jean-Claude Deshons, Philippe Jaulmes, Victor Vasarely, Albert Dupin, Robert Pillods, Fernand Michel, Yvaral (Jean-Pierre Vasarely), Yaacob Agam, Pol Bury, université, architecture universitaire, campus, Op-art, Art optique, Art cinétique, Montpellier

\section{AUTEUR}

\section{YVON COMTE}

Chargé d'études documentaires principal, chargé de la protection des monuments historiques à la conservation régionale des Monuments historiques de la direction régionale des Affaires culturelles d'Occitanie, ministère de la Culture yvon.comte@culture.gouv.fr 